\title{
Hipertensión pulmonar en la unidad de terapia intensiva
}

José Javier Elizalde González, ${ }^{*, \ddagger}$ Eli Omar Zavaleta Martínez ${ }^{\ddagger}$

Fue en 1891 cuando Ernst von Romberg describió en Alemania, en su libro Enfermedades del corazón y los vasos sanguíneos, la esclerosis de la arteria pulmonar sin una causa identificable; fue la primera referencia a la hipertensión pulmonar, compleja entidad que involucra muchas condiciones clínicas, puede complicar la mayoría de las cardiopatías y neumopatías avanzadas, y suele requerir un abordaje multidisciplinario. Se le divide por consenso en cinco diferentes grupos de acuerdo con sus características genéticas, histológicas, hemodinámicas, funcionales, clínicas y de respuesta terapéutica, entre otras (1: hipertensión arterial pulmonar [HAP], 2: producida por cardiopatía izquierda, 3: producida por neumopatía y/o hipoxia, 4: tromboembólica crónica, 5: con mecanismos multifactoriales y/o poco claros). En las últimas décadas, se han logrado avances extraordinarios en el entendimiento de su patobiología y fisiopatología, lo que ha dado lugar a la síntesis de nuevas moléculas que, de manera específica, actúan sobre el territorio vascular pulmonar.

Su epidemiología en el paciente mexicano se está describiendo actualmente a través de una importante iniciativa multiinstitucional contenida en el Registro Mexicano de Hipertensión Pulmonar (REMEHIP). ${ }^{1}$

No es infrecuente en el área de la medicina crítica enfrentarse a pacientes con este problema; sin embargo, suelen confundirse en nuestro medio el caso de un paciente con diagnóstico conocido de HAP (grupo 1) por un abordaje diagnóstico previo (que incluye, entre otros, un cateterismo derecho) y que sufre en forma aguda algún problema grave sobreagregado que lo lleva a la sala de intensivos en estado crítico, y ese otro paciente críticamente enfermo, a veces antes sano, que desarrolla en el curso de su padecimiento agudo hipertensión pulmonar (HP), muchas veces del grupo 2, y sobre todo del 3 , como puede ser el caso de un paciente con síndrome de insuficiencia respiratoria progresiva aguda (SIRPA). Ñamendys y sus colaboradores abordan en su trabajo un grupo de dichos pacientes, manejados en la unidad de terapia intensiva (UTI) del Instituto Nacional de Ciencias Médicas y Nutrición «Salvador Zubirán», con una prevalencia de $46.6 \%$ y sin impacto particular sobre la mortalidad (36.6\%) de esta dificultad respiratoria ya de por sí catastrófica; en ellos, el nivel de la presión de la vía aérea parece haber influenciado el inicio de la HP.²

\footnotetext{
* Editor.

‡ INCMNSZ.
}

Este artículo puede ser consultado en versión completa en http://www.medigraphic.com/medicinacritica
Esta confusión no es problema menor, dado que lleva generalmente, ante la incorrecta selección de casos, a la simplista prescripción de fármacos vasodilatadores pulmonares específicos no indicados en la mayor parte de los pacientes en estado crítico, en especial en la forma de una antifosfodiesterasa-5 del tipo del sildenafil, óxido nítrico inhalado o, en ocasiones, algún medicamento antiendotelina - problema que seguimos observando con preocupante frecuencia en distintas UTI a lo largo y ancho del país-. El Colegio Mexicano de Medicina Crítica (COMMEC) desarrolló hace pocos años un importante simposio sobre el particular, en parte con la finalidad de aclarar ciertos conceptos relacionados con este problema, aunque el mismo es obvio que persiste, quizá tanto por falta de un mayor conocimiento sobre el particular como por el bombardeo comercial y la presión que la industria suele hacer sobre los clínicos.

Como sabemos, la HAP es una enfermedad progresiva caracterizada por una vasculopatía que resulta en una elevación sostenida de la presión de la arteria pulmonar y que lleva, al final, a disfunción y falla del ventrículo derecho y, eventualmente, a la muerte. La HP del paciente, crítico aunque con algunas coincidencias, es una entidad distinta. Manejar de manera correcta a un enfermo agudo con HAP es todo un reto: sus mecanismos de falla ventricular derecha están entendidos de forma incompleta, no tolera bien cargas de líquidos y no existen suficientes datos provenientes de ensayos controlados para evaluar la superioridad de ciertos abordajes terapéuticos, además de problemas agregados, que suelen incluir en la UTI: órdenes de nada por vía oral, falla renal y/o hepática, alteración del estado de consciencia, delirio, inestabilidad hemodinámica con hipotensión arterial sistémica y sepsis.

Por ello, es necesario que el personal médico de la UTI amplíe sus conocimientos sobre el tema y solicite oportunamente la opinión del experto en hipertensión pulmonar; las decisiones de cómo manejar drogas vasodilatadoras pulmonares específicas pueden tener un impacto mayor sobre la evolución de estos enfermos.

La HP producida por cardiopatía izquierda no tiene a la fecha un manejo específico; dependiendo de la causa subyacente y con un nivel de recomendación IC, medidas como el correcto uso de drogas (del tipo de los diuréticos, nitratos, hidralazina, inhibidores de la enzima convertidora de la angiotensina, betabloqueadores, nesiritida, inotrópicos) o intervenciones como la asistencia ventricular izquierda, cirugía valvular, resincronización y trasplante cardiaco pueden lograr una reducción significativa de la presión pulmonar de manera más o menos rápida, a tra- 
vés de la disminución de la presión de llenado del ventrículo izquierdo. La HP producida por neumopatía y/o hipoxia requiere el uso de $\mathrm{O}_{2}$ suplementario (recomendación IC); en estos pacientes, el empleo de vasodilatadores puede producir un mayor grado de hipoxemia al romperse la vasoconstricción hipóxica. La experiencia publicada con vasodilatadores específicos es muy escasa, por lo que su utilización no está indicada (recomendación IIIC). Finalmente, el tratamiento de elección para la hipertensión pulmonar tromboembólica crónica es el quirúrgi$\mathrm{co}$, en la forma de una endarterectomía pulmonar por un grupo quirúrgico con experiencia en el procedimiento, no el tratamiento farmacológico de entrada.

La principal función del aparato respiratorio es el intercambio gaseoso; esto se lleva a cabo en un sistema circulatorio de baja resistencia. La circulación pulmonar constituye un sistema único que difiere substancialmente del sistémico (coronario, renal, cerebral, etcétera), tanto en estructura y función como en mecanismos de regulación, lo que el médico intensivista no debe olvidar en beneficio de sus pacientes en estado crítico.

\section{BIBLIOGRAFÍA}

1. Sandoval-Zarate J, Jerjes-Sanchez C, Ramirez-Rivera A, Zamudio TP, Gutierrez-Fajardo P, Elizalde-Gonzalez J, et al. Mexican registry of pulmonary hypertension: REMEHIP. Arch Cardiol Mex. 2017;87(1):13-17.

2. Ñamendys-Silva SA, Santos-Martínez LE, Pulido T, RiveroSigarroa E, Baltazar-Torres JA, Domínguez-Cherit G, et al. Pulmonary hypertension due to acute respiratory distress syndrome. Braz J Med Biol Res. 2014;47(10):904-910. 